\title{
Parques de bolsillo: un análisis desde la percepción de usuarios en la ciudad de México
}

\section{Pocket parks: an analysis from the perception of users in Mexico city}

\author{
Laura Elena Martínez Salvador* \\ David Fabricio Alvarado Ramírez**
}

\begin{abstract}
Pocket parks in Mexico City have been established as alternatives for areas recovery, which we propose to conceptualize as underused public spaces; the aim of this research is to analyze the case of three pocket parks through a methodology that integrates the perception that users have of the parks in terms of security, infrastructure, maintenance and mobility.
\end{abstract}

Keywords: public spaces, parks, city.

\section{Resumen}

Los parques de bolsillo en la Ciudad de México se han instaurado como alternativas de recuperación de áreas, los cuales proponemos conceptualizar como espacios públicos subutilizados; esta investigación tiene como objetivo principal analizar el caso de tres parques de bolsillo a través de una metodología que integre la percepción que los usuarios tienen de los parques en cuestión de seguridad, infraestructura, mantenimiento y movilidad.

Palabras clave: espacio público, parques, ciudad.

\footnotetext{
* Instituto de Investigaciones Sociales de la Universidad Nacional Autónoma de México, correo-e: laura.martinez@sociales.unam.mx

** Centro Interdisciplinario de Investigaciones y Estudios sobre Medio Ambiente y Desarrollo del Instituto Politécnico Nacional, correo-e: david.al.rmz@hotmail.com
} 


\section{Introducción}

Los espacios públicos pueden generar impactos positivos o negativos en la dinámica de las ciudades, lo cual depende, entre otras cosas, de la intervención de instituciones públicas como elemento indispensable en la reconversión de estos sitios para que los habitantes de la ciudad cuenten con lugares que les permitan desarrollar actividades de recreación, descanso y convivencia, en un ambiente de seguridad y protección, "[mejorando] el valor social y económico de los espacios intervenidos y la calidad de vida de los habitantes [de una ciudad]" (Quiroga, 2017: 8). Se pretende que el aprovechamiento y reconocimiento de los lugares recuperados en la ciudad facilite y estimule el acceso, la convivencia, la creación de dinámicas sociales positivas y que motive a los usuarios a aprovechar los espacios que, hasta ahora -ya sea por el limitado acceso o mal aspecto- no han captado su atención.

En la Ciudad de México se han generado estrategias de recuperación de espacios públicos que se encuentran subutilizados, tales como aquéllas emprendidas en el marco del Proyecto de Parques Públicos de Bolsillo, en adelante PPpb, diseñado por la Secretaria de Desarrollo Urbano y Vivienda de la Ciudad de México (Seduvi), en coordinación con el Gobierno del Distrito Federal, la Procuraduría Ambiental y del Ordenamiento Territorial (PAOT), y la Agencia del Espacio Público (AEP).

Estos esfuerzos de recuperación de los espacios públicos subutilizados constituyen, en sentido estricto, una estrategia para incidir en la funcionalidad de los lugares y generar efectos positivos en la percepción que la población tiene de los mismos incentivando su aprovechamiento; las estrategias de recuperación de estos lugares, como el PPpb, poseen en su naturaleza misma y en sus lineamientos institucionales, la búsqueda de la mejora de las condiciones de vida de los usuarios, pero con planteamientos que podrían mejorarla aunque de forma solo tangencial. Sin embargo, la ausencia de vinculación entre instituciones participantes, así como la falta de reglas de operación y términos de referencia oficiales en torno a los proyectos, dificultan la implementación y mantenimiento de los espacios, dando como resultado espacios recuperados deficientes con efectos limitados.

El objetivo general del presente artículo fue analizar el caso de tres parques públicos de bolsillo diseñados en el marco del PPpb y discernir si éstos pueden considerarse como alternativa para la recuperación de espacios públicos subutilizados, el análisis se realizó mediante una metodología que integró la percepción que los usuarios tienen del parque en cuestión de cuatro ejes: seguridad, infraestructura, mantenimiento y movilidad. 
Se diseñó una herramienta que identificó la percepción de los usuarios a propósito de los parques de bolsillo desde los ejes mencionados; lo anterior se complementó con una serie de visitas de campo a los tres parques públicos de bolsillo, los cuales fueron elegidos por sus características y relevancia en los entornos seleccionados. Finalmente, se desarrolló un análisis crítico de las debilidades y oportunidades de dichos parques públicos de bolsillo; como conclusión se integraron algunas reflexiones generales y recomendaciones para la mejora de estas estrategias de recuperación de espacios públicos subutilizados.

\section{Estado del arte y la discusión actual de los espacios públicos subutilizados}

Dentro de las concepciones en torno al concepto de espacio público encontramos múltiples visiones, sin embargo, existen puntos convergentes bajo los cuales es posible construir conocimiento relevante para el análisis de casos específicos.

En principio podemos partir de la concepción de Carrión (2016: 18) respecto al espacio público, entendiéndolo como "lo que queda, como lo residual, como lo marginal después de construir vivienda, comercio o administración"; esta definición, la cual puede parecer un tanto limitada debido a su ambigüedad, nos permite plantear que el espacio público puede estar compuesto por vialidades y lugares con usos tan diversos como la recreación y el esparcimiento, y permite a su vez desarrollar nodos de intercambios de conocimiento, bienes e incluso determinar símbolos urbanos.

Acorde con la anterior reflexión es importante destacar que los espacios públicos, al presentarse en esquemas de vialidades principales - que TerrazasRevilla (2005) considera "esquema de ejes y caminos"- tienen amplio potencial para generar efectos positivos y bienestar en la población transeúnte, especialmente si las ubicaciones favorecen la movilidad tanto de vehículos como de peatones; no obstante, para ello se requiere un óptimo aprovechamiento de sus características básicas y del potencial de desarrollo.

En este espacio público se vislumbra una serie de signos que permiten generar identidad en el territorio y pueden, en ocasiones, generar rechazo o aceptación por el espacio (Checa-Artasu, 2017). Por otra parte, para Subirats (2016) los espacios públicos generan relaciones y vinculaciones de distintos actores que poseen condiciones sociales y personales distintas, derivadas, a su vez, de la diversidad de sus recursos financieros, personales o emocionales.

Los diversos tipos de usuarios que convergen en estos espacios públicos generan flujos de ocupación que cambian a lo largo del tiempo. Sin 
embargo, a pesar de que estos enfoques mantienen importancia en la unificación del colectivo en los espacios públicos, la heterogeneidad de los actores constituye una de las principales fortalezas de éstos, pero también uno de los principales retos.

Otras formas de entender los espacios públicos es a través de la concepción de que son sitios físicos: calles, plazuelas, parques y áreas verdes de interés general para la ciudad "donde se desvanece la individualidad y se transita de lo privado a o público" (Rabotnikof, 2010; León, 1998: 19), lugares que presentan características socioculturales definidas y constituyen sitios de encuentro que, asimismo, fungen como indicadores de la calidad urbana (Borja y Muxi, 2000).

Estos ejemplos de espacios físicos se definen por ser abiertos y accesibles a todos, lugares de libertad e inclusión que favorecen la vinculación social y las prácticas comunitarias. En esta idea, es menester hacer énfasis en que aquellos espacios públicos deficientes en la formación de vínculos entre actores sociales, así como zonas recreativas y de esparcimiento con evidentes muestras de descuido, pueden ser considerados espacios públicos que permanecen en desuso o incluso vacíos.

Para Bellet Sanfeliu y Llop Torné (2004) existen espacios en desuso con equipamientos e infraestructuras en condición de abandono, los cuales son vistos como "vacíos urbanos" o, como menciona Curzio (2008: 55), como territorios residuales que "pueden ser la porción derivada de una obra mayor o el resultante de la transformación destructiva originada por el paso del tiempo", estos vacíos pueden referirse a antiguas zonas industriales, instalaciones ferroviarias o instalaciones militares objeto de recuperación a través de proyectos que buscan reconstruir sus funcionalidades y que se transforman en espacios de equipamiento urbano.

Fausto y Rábago (2001) e Iracheta (2014) hacen reflexiones en torno a los vacíos urbanos, establecen que son aquellos espacios construidos con potencial de ser utilizables, o cuyo uso sea evidentemente inferior a su potencial de aprovechamiento urbano,que se localizan primordialmente en el interior de áreas urbanizadas; para Clichevsky (2007) el espacio del que no se puede definir su estado actual, pero cuyo desaprovechamiento los vuelve como tierra vacante no utilizada, es aquélla que se encuentra: "subdividida en parcelas denominadas urbanas según la legislación vigente, dentro del perímetro de la aglomeración, y que podrían ser usadas para fines residenciales, industriales, comerciales y de servicios" (Clichevsky, 2007: 198).

Con base en las reflexiones anteriores, se propone la conceptualización de los espacios públicos subutilizados como aquellos lugares cuyo uso actual colectivo es evidentemente inferior a su potencial de aprovechamiento y cuya recuperación puede ser con fines culturales, recreacionales, 
comerciales o de servicios; dichos espacios públicos subutilizados deben poseer características físicas, geográficas y administrativas idóneas para su recuperación y transformación en la búsqueda de generar lugares funcionales y adecuados que impulsen la convivencia y la creación de identidad comunitaria.

El potencial que los espacios subutilizados tienen para aportar bienestar a la población depende no solo de acciones de recuperación, sino también de estrategias de apropiación y planes de mantenimiento, las acciones de recuperación generadas en la planeación urbana pueden tener mayor porcentaje de éxito y apropiación si en su diseño se considera al territorio mismo, especialmente las necesidades que los usuarios puedan tener (Bratina Jurkovic, 2014); ejercicios como el desarrollado en el marco de esta investigación son relevantes en la construcción de proyectos de recuperación basados en intereses reales de la población.

\section{Los espacios públicos subutilizados en la Ciudad de México. Alternativas de recuperación}

Una vez definidos los espacios públicos subutilizados, podemos establecer que en la Ciudad de México cohabitan locaciones plagadas de edificios, plazas, parques, jardines públicos y privados, así como espacios que pueden apreciarse en aparente estado de abandono. El espectador urbano puede catalogarlos como subutilizados, ya que su potencial de aprovechamiento no está adecuadamente desarrollado; estos espacios en ocasiones se perciben también como lugares de riesgo para el usuario, debido a que son utilizados como basureros clandestinos, con vegetación y fauna nociva o, incluso, como viviendas temporales o permanentes por personas en situación de calle.

En algunos casos son los mismos usuarios quienes ocupan estos espacios de manera inadecuada, de forma tal que "lo urbano ya no es un lugar que garantiza la integración social [...] ya que no fomenta la proximidad ni las relaciones ni los encuentros sociales" (Delgadillo, 2016: 142). De lo anterior se desprende que el patrimonio público puede verse demeritado por ciudadanos, quienes no utilizan las zonas públicas como centros de convivencia.

Ante la mala condición o deterioro de la infraestructura de algunos de estos espacios, se han hecho propuestas para mejorarlos visualmente, invitando así a los usuarios a visitarlos, recorrerlos, utilizarlos y disfrutarlos, de forma tal que se brinde un lugar de descanso donde sea posible incentivar buenas prácticas sociales. De la misma manera y ante la colocación de equipamiento novedoso y atractivo se busca que se desarrollen actividades al aire libre para crear un espacio en el que los habitantes de 
la ciudad se sientan cómodos; sin embargo, para ello se requieren de procesos de rehabilitación del espacio público.

En este sentido, "la rehabilitación del espacio público impulsa la reapropiación de la calle como elemento central para la cohesión social siendo que el mejoramiento urbano ha comenzado a extenderse hacia los inmuebles y procura la ocupación" (GDF, 2011: 110). Las zonas recuperadas corresponden a espacios públicos como patios, banquetas, plazas, calles, entre otros, que, habiendo perdido la función contemplada para su uso desde el comienzo de su operación, permanecían subutilizadas o carentes de infraestructura adecuada para su aprovechamiento.

Ante este escenario, la búsqueda de la sostenibilidad urbana ha dado paso a alternativas de transformación de estos espacios urbanos a zonas verdes o recreativas para el mejoramiento visual, el desarrollo ambiental y social de la comunidad. Estos lugares aparentemente subutilizados son la representación de una ciudad que necesita un cambio en su forma de interactuar con su medio, por ello es necesario considerar proyectos de recuperación: los habitantes de la ciudad tienen derecho a contar con zonas que les permitan desarrollar actividades recreacionales y que no se encuentren tan alejados de sus lugares de vivienda.

\subsection{El Proyecto Parques Públicos de Bolsillo en la Ciudad de}

\section{México}

Para el rescate de espacios públicos subutilizados es necesario contar con un marco normativo, institucional y operativo adecuado que justifique la acción y la posterior instauración de infraestructura como parques de bolsillo. Si existen los elementos normativos y los instrumentos o mecanismos de carácter público es posible generar evaluaciones a fin de conocer los efectos de las implementaciones y mejoras que los proyectos tienen en las zonas y en los usuarios objetivos. En la administración pública se conforman proyectos que son parte de programas insertos en marcos normativos vigentes, usualmente alineados a planes nacionales de desarrollo y materializados dentro de documentos oficiales expedidos, usualmente y para efectos locales, por el Gobierno de la ciudad de México.

En la búsqueda de un óptimo aprovechamiento de los espacios públicos subutilizados, el Gobierno de la Ciudad de México implementó en 2014 un programa de recopilación de opiniones colectivas para el direccionamiento de recursos públicos, este programa denominado "Decisiones por colonia" tenía por objetivo identificar las necesidades prioritarias de la ciudadanía en múltiples áreas, una de ellas era la atención a los espacios públicos. Si bien el ejercicio de democratización del recurso público dejó entrever muchas necesidades de la urbe, y aun cuando podríamos analizar 
el ejercicio de participación ciudadana desde una perspectiva más crítica, uno de los resultados de esta consulta ciudadana fue la necesidad imperiosa de recuperar aquellos espacios que se encontraban en desuso (Seduvi, 2014: 5).

Ante la integración de opiniones ciudadanas se plantearon estrategias para dar a los espacios subutilizados una segunda oportunidad con funciones recreativas y de impulso el capital social, que favoreciera la peatonalización de las zonas recuperadas, el mejoramiento de la imagen urbana, e incluso que propusiera, aunque sin grandes argumentaciones, la disminución de la huella ecológica.

Es importante destacar que, en la idea de rescatar espacios, quienes toman decisiones sobre el destino de una ciudad pueden optar por distintas alternativas de recuperación de espacios públicos; una de ellas puede ser la implementación de parques de bolsillo, bajo este supuesto surgió el PPpb en la Ciudad de México, un proyecto que retomaba ideas del sistema de parques del Distrito Federal desarrollado por la Secretaría del Medio Ambiente (Sedema, s/f), en el cual se definían a los parques públicos como:

espacios verdes de menor tamaño que contribuyen a regular el clima urbano [...] generando equilibrios ambientales en suelo, agua y aire. Estos parques son fundamentales para el esparcimiento, la recreación y el reforzamiento de la identidad de barrios y colonias en el entorno urbano.

Así mismo, se categorizaba a los parques públicos de la Ciudad de México en cinco tipos con base en sus dimensiones: metropolitanos, locales, barriales, lineales y públicos de bolsillo (Seduvi, 2014). En 2014, la Seduvi definió por primera vez a los parques públicos de bolsillo como: “pequeños espacios en remanentes, urbanos o viales, subutilizados o abandonados [que son] convertidos en espacios para la recreación y disfrute de la comunidad" (Seduvi, 2014: 14-16).

Estos parques se caracterizan por sus pequeńas dimensiones, entre 100 y $400 \mathrm{~m}^{2}$, y porque tienen como objetivo principal aportar a la mejora de la calidad de vida de los usuarios, así como a la imagen urbana de la zona, debían contribuir a la creación de comunidad y a la mejora de la calidad de vida, lo que debía estar aparejado con un impulso a la seguridad vial, la instauración de cruces seguros - con el peatón como elemento principaly a un mejoramiento de la salud pública de ciudades altamente pobladas como la Ciudad de México (Seduvi, 2014).

Al conocerse los resultados de la consulta ciudadana, la Seduvi y la Procuraduría Ambiental y del Ordenamiento Territorial (PAOT) definieron el PPpb con base en el documento "Lineamientos para el diseño e implementación de parques públicos de bolsillo", más no un programa oficial 
institucionalizado. En el planteamiento del documento guía para la instauración de parques de bolsillo se carecía de objetivos asequibles, medibles, alcanzables y realizables; de igual forma, no se contaba con indicadores de temporalidad ni de resultados, no se encontraron reglas de operación que determinaran las dimensiones, el lugar, la infraestructura y demás especificaciones que se necesitaban para tener un espacio público rescatado y con las características que promovieran su uso por parte de la comunidad.

Al no contar con ejes articuladores ni con el componente de políticas públicas (que implica la alineación del PPpb con algún programa de rescate de espacios, local o regional) no fue posible apreciar la inclusión de este proyecto en programas como el Programa de rescate de espacios públicos de la Secretaría de Desarrollo Agrario, Territorial y Urbano, el Programa sectorial de desarrollo agrario, territorial y urbano 2013-2018, el Programa general de desarrollo del distrito federal 2013-2018 o el Subprograma integral de movilidad 2013-2018, normativa encargada de dar arranque, evaluación y mantenimiento a mecanismos de recuperación de espacios urbanos.

Por lo tanto, el PPpb como instrumento de política pública podría considerarse insuficiente para la instauración de acciones de rescate urbano o para la evaluación de proyectos de carácter público; surge también la reflexión en torno a las inconsistencias que valdría la pena subsanar en futuros ejercicios de gobernanza participativa y en la definición de la metodología seguida en los foros de participación ciudadana.

Es importante destacar que crear mecanismos que promuevan la participación ciudadana es siempre una estrategia de democratización de las decisiones, pero la carencia de transparencia metodológica y de resultados puede generar incertidumbre en cuanto a la elaboración de una o varias estrategias consensuadas.

\section{Metodología}

La metodología seguida para el desarrollo de esta investigación estuvo basada en una revisión literaria, así como en un análisis epistemológico para la construcción de una propuesta conceptual que defina espacios públicos subutilizados, de forma tal que puedan integrarse visiones diversas del desuso urbano. Posteriormente, se seleccionó una serie de casos de estudio de espacios en situación de subutilización con base en el aporte conceptual propuesto y con una reconversión bajo el PPpb.

De los 35 parques de bolsillo localizados en la Ciudad de México (AEP, 2018) se seleccionaron los siguientes para el análisis del nivel de percepción 
que, en materia de seguridad, infraestructura, mantenimiento y movilidad del entorno, tienen los usuarios: Parque de Bolsillo El Rosario, Parque de Bolsillo Tezozómoc (ambos en la alcaldía Azcapotzalco) y Parque de Bolsillo Zócalo (en la alcaldía Cuauhtémoc).

Los casos de estudio se seleccionaron de manera estratificada, esto implicó ubicar las alcaldías con la mayor densidad de parques de bolsillo, lo que denotó una vocación más amplia a implementar este tipo de proyectos, las alcaldías Cuauhtémoc y Azcapotzalco concentraban 37 por ciento de los parques instaurados.

En estas alcaldías se seleccionaron los casos con base en los siguientes justificantes socioterritoriales: el parque Zócalo se seleccionó porque fue el primero en su tipo desarrollado en la Ciudad de México en 2013 (Sosa, 2013), analizarlo permitirá conocer los resultados del primer ejercicio de recuperación de espacios públicos subutilizados desde el inicio del PPpb. El parque de bolsillo Tezozómoc fue seleccionado por ser el más grande en la Ciudad de México, con lo que se favorece el conocimiento en torno a uno de los espacios con la mayor dimensión de aprovechamiento, mientras que el parque de bolsillo El Rosario fue elegido por ser el único ubicado al interior de una de las unidades habitacionales más grandes y representativas de América Latina (ALDF, 2016), la Unidad Habitacional El Rosario. Esto nos permite conocer las condiciones del parque y sus efectos en una de las ubicaciones con mayor densidad poblacional.

La siguiente etapa del trabajo de investigación consistió en realizar una serie de visitas de campo a los espacios públicos subutilizados y recuperados bajo el marco del PPpb, aquí se identificaron las características del entorno inmediato del parque de bolsillo: la ubicación geográfica, representación de calles, elementos del entorno ecológico, elementos de flujo en la movilidad peatonal y vehicular, señalización de equipamientos diversos, usos del espacio y tipo de usuarios, así como algunas cuestiones sensoriales del espacio y la imagen urbana.

Posteriormente se diseńó un instrumento de análisis para el diagnóstico del estado actual de percepción de los usuarios de los parques de bolsillo en materia de seguridad, infraestructura, limpieza, mantenimiento e incluso ubicación, éste se operacionalizó a través de un cuestionario. En esta misma etapa se hizo un ejercicio de encuesta no probabilística limitada a 15 personas usuarias, quienes tenían un momento de consumo en locales o utilizaban la infraestructura recuperada; 50 por ciento de los encuestados eran mujeres y el resto eran hombres, con edades entre 25 y 45 años.

Es importante mencionar que, aunque la recopilación de información por encuestas mencionada posee limitantes a nivel estadístico y de representatividad muestral, este ejercicio de análisis de la percepción de usuarios contribuye a construir de forma más completa una evaluación crítica en 
torno al actual estado de los espacios recuperados y proporciona una visión de las necesidades más apremiantes a ser atendidas, a razón de las necesidades del usuario final.

Por otra parte, considerando la ausencia de un padrón confiable que especifique el número de usuarios que visitan los espacios, no fue posible definir una muestra estadísticamente representativa. Además, la reticencia y desconfianza del usuario promedio constituyó la principal barrera para la aplicación de encuestas.

En una siguiente etapa la recopilación de información de las visitas in situ, así como la información cuantitativa de la encuesta, fue tratada y analizada a través de gráficas de radiales y de un análisis siguiendo la metodología estudio de caso (Yin, 2009).

\subsection{Construcción de un instrumento de análisis para el diagnóstico del estado actual de percepción de los usuarios de parques de bolsillo}

En la Ciudad de México, los esfuerzos en materia de evaluación de proyectos de recuperación de espacios urbanos son incipientes; uno de los trabajos más destacados lo desarrolló la AEP (2018), en éste, a través de un sondeo, se determinó el nivel de satisfacción de usuarios respecto a la calidad de la infraestructura de algunos parques de bolsillo. En los resultados de este ejercicio se encontró que estos lugares son en su mayoría espacios de paso y no constituyen un destino en sí, ya que las actividades principales son de tránsito peatonal, por lo tanto, no crean identidad barrial ni llegan a ser un hito importante dentro del ambiente urbano. No obstante, esta investigación posee limitantes, principalmente porque no incluye otros elementos en el análisis como la percepción en materia de seguridad, movilidad, estado del mantenimiento e incluso pertinencia de la ubicación.

Considerando lo anterior, y a fin de identificar la percepción de los usuarios en torno al estado actual de los parques públicos de bolsillo se generó una herramienta de diagnóstico basada en un cuestionario con la escala de percepción: excelente 3 puntos; bueno 2 puntos; regular 1 punto; pésimo 0 puntos; se consideraron los siguientes elementos: ubicación, seguridad, infraestructura, mantenimiento y condiciones de movilidad. El contenido puede ser visto en la tabla 1.

Esta herramienta se aplicó en forma de cuestionario a usuarios de los parques de bolsillo. En los siguientes apartados se presenta el diagnóstico haciendo uso de diagramas radiales y puntualizando las características más relevantes de cada espacio seleccionado. La aplicación de las encuestas se realizó de forma aleatoria. 


\section{Tabla 1}

Elementos de diagnóstico del estado actual de percepción del usuario de parques públicos de bolsillo

\begin{tabular}{|c|c|}
\hline \multicolumn{2}{|c|}{ Parque Público de Bolsillo (Nombre del parque público de bolsillo): } \\
\hline Sexo: M( ) F ( ) Edad: & $\begin{array}{l}\text { ESCALA DE PERCEPCIÓN: Excelente (3); Bueno (2); } \\
\text { Regular (1); Pésimo (0) }\end{array}$ \\
\hline \multicolumn{2}{|c|}{ ELEMENTO DE ANÁLISIS } \\
\hline \multirow{3}{*}{ UBICACIÓN } & Localización \\
\hline & Tiendas y/o restaurantes \\
\hline & Suma parcial \\
\hline \multirow{3}{*}{ SEGURIDAD } & Nivel de seguridad percibido \\
\hline & Iluminación \\
\hline & Suma parcial \\
\hline \multirow{8}{*}{ INFRAESTRUCTURA } & Vegetación y espacios verdes \\
\hline & Bebederos \\
\hline & Nivel de comodidad de la infraestructura \\
\hline & Aparcamiento bicicletas \\
\hline & Área recreativa infantil \\
\hline & Área de acondicionamiento físico \\
\hline & Internet (wi-fi) \\
\hline & Suma parcial \\
\hline \multirow{4}{*}{ MANTENIMIENTO } & Estado general de mantenimiento \\
\hline & Limpieza de las áreas \\
\hline & Contaminación periférica \\
\hline & Suma parcial \\
\hline \multirow{2}{*}{ MOVILIDAD } & Accesos y rampas para mayor movilidad \\
\hline & Suma parcial \\
\hline \multicolumn{2}{|l|}{ SUMA TOTAL } \\
\hline \multicolumn{2}{|l|}{ PROMEDIO } \\
\hline
\end{tabular}

Fuente: elaboración propia. 


\section{Diagnóstico de los parques de bolsillo en la Ciudad de México}

\subsection{Parque Público de Bolsillo Tezozómoc, Azcapotzalco, Ciudad de México}

El parque de bolsillo Tezozómoc puede considerarse, en sentido estricto, un parque barrial por sus dimensiones $\left(720 \mathrm{~m}^{2}\right)$, sin embargo, fue incluido como parte de los parques de bolsillo desde el inicio del proyecto. Se localiza en la alcaldía Azcapotzalco en la Ciudad de México, está ubicado frente la estación del Sistema de Transporte Colectivo, Metro Tezozómoc, cerca de conjuntos habitacionales; tiene acceso colindante por la avenida principal Parque Vía.

Por ser el parque de bolsillo más grande en construirse, cuenta con casi todos los tipos de vegetación que propone la guía de lineamientos mencionada con anterioridad (Seduvi, 2014). El parque Tezozómoc cuenta con acceso para personas con capacidades diferentes: rampas para sillas de ruedas, guías videntes y tableros con información de las distintas ubicaciones en Braille. Cuenta con arbolado ornamental dentro de la infraestructura de descanso, también con vegetación a nivel del suelo, instalaciones de área infantil, así como infraestructura utilizada para patinetas (por ello a este parque se le conoce como Skatepark Tezozómoc). En sus instalaciones existe también una fuente brotante con función programada y postes de luz solares. En cuanto al análisis del estado actual de percepción de los usuarios del parque de bolsillo, la gráfica 1 muestra los resultados.

El parque Tezozómoc es considerado como uno con buena infraestructura, como puede observarse en la gráfica 1, tres de los cinco encuestados calificaron como excelente este rubro. Dada la cercanía con el STC metro Tezozómoc, este espacio está muy bien ubicado y cuenta con múltiples servicios de alimentos cercanos. Por otra parte, la percepción de seguridad también es positiva, ya que cuenta con buena y constante iluminación. 


\section{Gráfica 1}

\section{Elementos de diagnóstico del estado actual de percepción del usuario en el parque de bolsillo Tezozómoc}

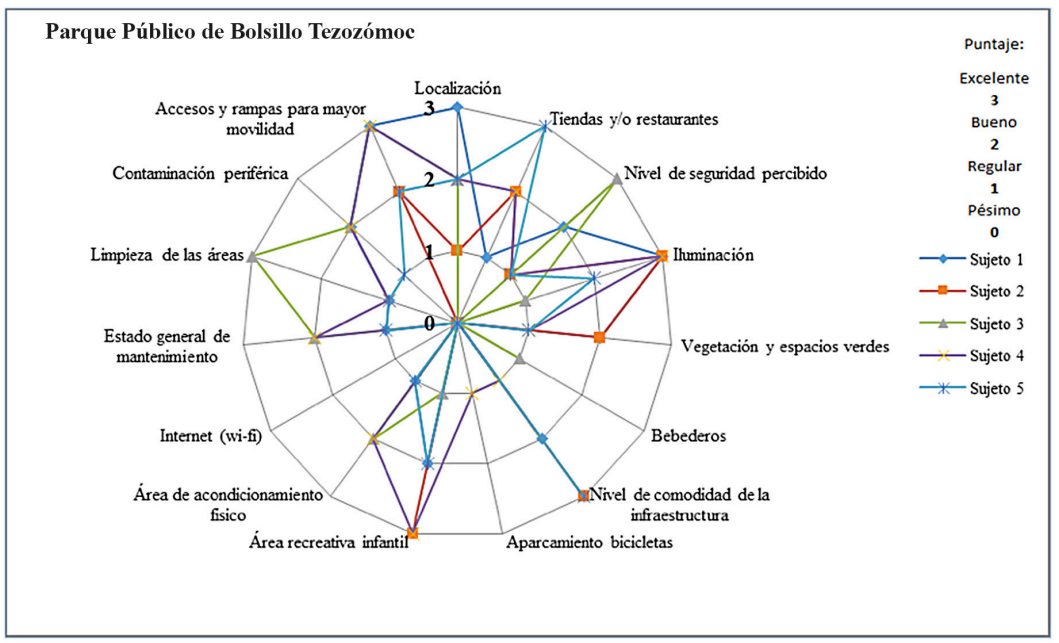

Fuente: elaboración propia con base en encuestas realizadas.

\subsection{Parque Público de Bolsillo Zócalo, Cuaubtémoc, Ciudad de México}

El parque Zócalo tiene una dimensión de $245 \mathrm{~m}^{2}$ aproximadamente y se ubica en la alcaldía Cuauhtémoc, Ciudad de México, México, fue el primero de su tipo en inaugurarse en la ciudad y contiene la mayoría de las instalaciones de descanso y recreativas necesarias; cuenta con mesas de acero y bancas con maceteros como respaldo, vegetación a nivel de piso y arbustos ornamentales, área de juegos infantiles y piso anti-derrapante, bebederos y área para mantenimiento de bicicletas, además de bolardos e iluminación con postes de luz fría. En cuanto al diagnóstico del estado actual, la gráfica 2 integra los elementos de diagnóstico.

Como puede observarse en la gráfica 2, la iluminación ayuda a la percepción de seguridad, asimismo, el mantenimiento que se ha realizado a bebederos y a la vegetación del espacio ha creado una sensación de comodidad. Este parque tiene una ubicación cerca de tiendas y cuenta con áreas de descanso, así mismo, cuenta con elementos de movilidad adecuados, aunque la cercanía con el paso de vehículos favorece la contaminación periférica. 


\section{Gráfica 2 \\ Elementos de diagnóstico del estado actual de percepción del usuario en el parque de bolsillo Zócalo}

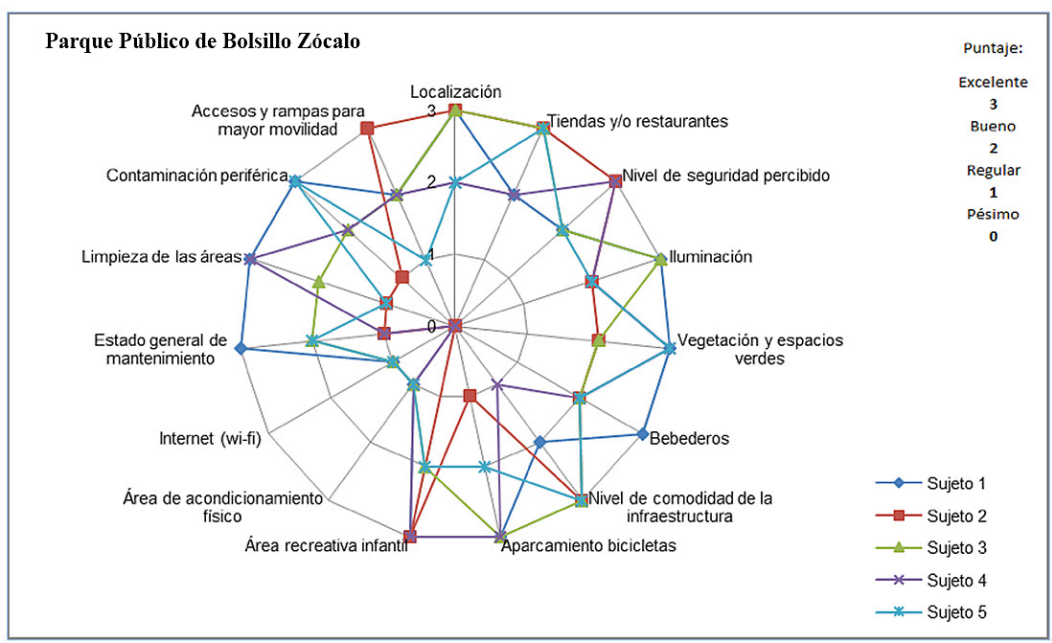

Fuente: elaboración propia con base en encuestas realizadas.

\subsection{Parque Público de Bolsillo El Rosario, Azcapotzalco, Ciudad de México}

El parque de bolsillo El Rosario tiene una dimensión aproximada de $312 \mathrm{~m}^{2}$ y se ubica en la alcaldía de Azcapotzalco, Ciudad de México, México. Está ubicado en un área habitacional, cercano a una escuela secundaria; cuenta con área de juegos infantiles tubulares, muy diferentes a los de plástico de alta densidad de otros parques de la ciudad. Dentro de este espacio hay arbustos y árboles que requieren poco mantenimiento (únicamente poda y riego). Llama la atención la existencia de un "muro verde" con sistema de riego autónomo funcional tanto para temporadas de lluvia como para mantenimiento. En cuanto al diagnóstico del estado actual de percepción de los usuarios encuestados, la gráfica 3 contiene los resultados.

Este parque está ubicado en una de las zonas habitacionales más grandes de América Latina en la actualidad y socialmente conflictiva por los altos índices de delincuencia, situación acorde con el bajo nivel de seguridad percibido a pesar de la iluminación. El estado general de mantenimiento se considera regular, hay abandono en la infraestructura; como es un parque localizado entre casas-habitación se favorece la buena percepción respecto al nivel de contaminación periférico, pero esta misma característica hace parecer que este espacio es solo para el disfrute de los locales, quienes tampoco 


\section{Gráfica 3}

\section{Elementos de diagnóstico del estado actual de percepción del} usuario en el parque de bolsillo El Rosario

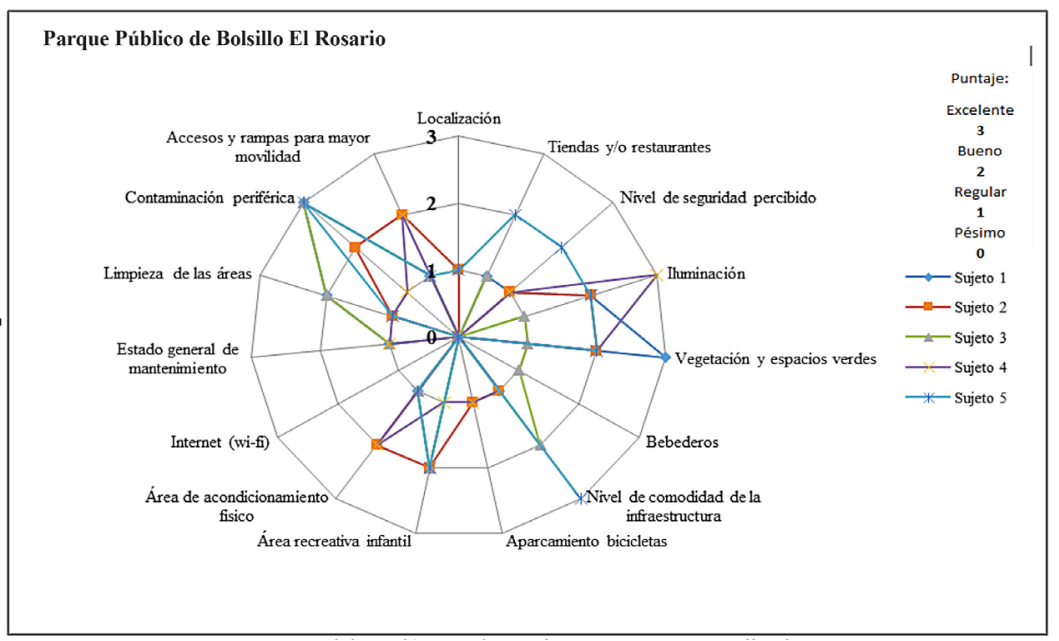

Fuente: elaboración propia con base en encuestas realizadas.

permanecen mucho tiempo en el lugar: la percepción general del parque no es favorable, la mayoría de las calificaciones oscilan entre pésimo y regular.

\section{Análisis de los parques públicos de bolsillo en la Ciudad de México. Discusión de resultados}

Los parques públicos de bolsillo analizados son lugares habitables para los usuarios, principalmente en los rubros de limpieza, movilidad comodidad, seguridad, iluminación, vegetación, áreas infantiles y de acondicionamiento físico. Al comparar los resultados de las tres series de encuestas, es claro que la percepción promedio es muy similar. Los usuarios han logrado acostumbrarse a transitar por estos espacios, aunque perciben poca mejoría en cuanto al entorno, producto de la instauración de estos espacios. De igual forma, la integración comunitaria entre los vecinos de los parques y las zonas recuperadas es un punto pendiente.

Para algunos usuarios, los parques públicos de bolsillo representan solo un punto de espera al intentar cruzar avenidas, calles o ejes viales. Es posible que no todos tomen en cuenta estos lugares, por lo que pasan de ser subutilizados recuperados, a lugares con infraestructura nueva, pero comúnmente en el olvido. Sin embargo, con propuestas ciudadanas de quienes utilizan y transitan por los espacios recuperados, reconociendo 
los principales aciertos y fallas de infraestructura, ubicación y movilidad que se requiere implementar, es posible impulsar acciones para que estos espacios cubran las expectativas de los usuarios actuales y futuros, generando una derrama de beneficios de esta infraestructura urbana.

Los parques públicos de bolsillo ofrecen versatilidad en instalaciones y equipamiento, tal es el caso de las atracciones que ofrecen a distintos sectores de la población, desde juegos infantiles hechos por rotomoldeo hasta pistas de patinetas, sin embargo, el mal uso, la falta de mantenimiento y el vandalismo disminuyen su calidad, uso y atractividad para quienes transitan diariamente por los parques.

Es posible que la falta de cultura cívica de la población, aunada a la falta de mantenimiento, acelere el deterioro de la infraestructura.También es importante mencionar que los espacios públicos, al ser lugares al exterior están en contacto con flujo peatonal, vehicular y condiciones climáticas como lluvia, sol o viento, así como factores antropogénicos, como el tránsito vehicular, choques, invasión de espacios peatonales en los parques, lo que puede acelerar el deterioro del mobiliario.

En el caso los parques públicos de bolsillo localizados entre vialidades -como el Zócalo- se presenta una condición en particular, ya que a pesar de las buenas intenciones de colocar contenedores vegetales (maceteros) y bolardos como medida preventiva de seguridad del peatón ante el tráfico vehicular, se percibe una sensación de incomodidad por encontrarse en un espacio constantemente rodeado por vehículos. Con estas implementaciones no se consigue elevar adecuada o suficientemente el índice de área verde por habitante, ya que la vegetación colocada es más ornamental que funcional.

La limpieza de los espacios es un factor que no siempre está presente; el constante flujo vehicular, el intenso paso peatonal y la falta de higiene se unen a la carencia de mantenimiento y cuidado de la salud del usuario. Por otra parte, una de las principales acciones que se llevaron a cabo para mejorar la seguridad, así como mejorar la imagen urbana y fortalecer la vigilancia, fue la de mantener una iluminación constante, aunque aún existen puntos donde ésta es deficiente.

Es importante recalcar que, si bien existen diversos manuales de movilidad, tránsito vehicular y peatonal -donde se describen diversos procesos para tomar en cuenta y tener una mejor movilidad dentro de la ciudadmuchos de estos manuales tienen contenido promocional gubernamental y carecen de mecanismos de aplicabilidad. Muchos son los que contienen exclusivamente logros alcanzados a corto plazo, pero no se cuenta con estudios que demuestren las limitaciones y problemas básicos de los espacios recuperados, como mantenimiento y destrucción de la infraestructura. 
En las visitas de reconocimiento a los parques de bolsillo se pudo apreciar que gran parte de los usuarios que utilizan el mobiliario urbano para los propósitos que fueron diseñados son nińos de entre 4 a 10 años, usualmente acompañados de padres de familia.

Con base en testimonios de los usuarios, se afirma que el poder observar a sus hijos realizando actividades en lugares que antes eran considerados peligrosos los motiva a su uso. Sin embargo, la cuestión es diferente en los territorios altamente poblados, como las unidades habitacionales donde se tienen identificadas zonas de alta incidencia delictiva como El Rosario, donde la percepción de seguridad no es muy elevada y, a pesar de contar con iluminación adecuada, los espacios carecen de vigilancia continua o botones de pánico.

Para hacer uso de la infraestructura pública recuperada y facilitar espacios de convivencia social y deportiva se ha implementado también, como medida de prevención, el intento de organizar a los jóvenes para su participación en actividades grupales y para formar parte de dinámicas barriales colectivas. Ante "la secuencia territorial distinta que presentan las metrópolis" (Terrazas-Revilla, 1995: 336) se requieren diversos proyectos de rescate de los espacios públicos acordes a las necesidades de cada territorio.

Es importante mencionar que la piedra angular de los programas de recuperación de espacios públicos subutilizados es la verdadera intención de usarlos conforme a su propósito, evitando malas prácticas. Sin embargo, la población carece de incentivos para hacer de estos espacios lugares más habitables y con una mejora en su morfología, por lo que quedan desatendidos dejándolos como un espacio más dentro de la ciudad con bajas aportaciones al ambiente ecológico y social.

Es desafortunado observar cómo estos lugares pierden su esencia con el paso del tiempo, ya que al contener equipamiento dedicado al descanso, los asientos son objetos de uso muy temporal; el constante uso de los espacios peatonales desgasta la pintura dando sensación de poca atención y deficiente mantenimiento, lo mismo ocurre con el equipamiento que es dedicado a conservar la integridad física del peatón tales como bolardos $\mathrm{y}$ aceras.

Una forma de impulsar el mejor uso de estos espacios es crear una adecuada señalización en torno al buen manejo de las instalaciones, así como una descripción de los beneficios para que al utilizar estos lugares se comprenda que son de uso público y para el disfrute de la sociedad. 


\section{Conclusiones}

Con base en esta investigación, podemos establecer que los objetivos del Proyecto de Parques Públicos de Bolsillo en la Ciudad de México definidos en sus lineamientos se cumplen en algunos aspectos y con efectos positivos marginales, esto quiere decir que a pesar de que la mejora del espacio es fácilmente apreciable, los efectos en el bienestar de la población representan una cuestión cualitativa más compleja de alcanzar, ya que depende de factores fuera del alcance de proyectos de esta naturaleza.

El PPpb posee en su naturaleza misma la búsqueda de la mejora de las condiciones de vida de los usuarios, sin embargo, la ausencia de vinculación entre instituciones participantes, así como la falta de reglas de operación y términos de referencia oficiales en torno a los proyectos de recuperación de espacios públicos subutilizados dificultan la instrumentación y mantenimiento, el resultado son espacios recuperados deficientes con efectos positivos limitados. Por lo tanto, el cumplimiento de los objetivos de estos proyectos de recuperación de espacios públicos en materia de ubicación, seguridad, infraestructura y movilidad es insuficiente.

Proyectos como el PPpb carecen de una normatividad oficial, reglas de operación y términos de referencia adecuados; consideramos que el establecimiento de los lineamientos alrededor de los convocantes, participantes y efectos esperados, así como demás elementos requeridos para ejercer acciones de política pública en materia de desarrollo urbano y recuperación de espacios públicos son inexistentes. Esto es preocupante, ya que estas acciones requieren de recursos públicos $y$, aun cuando tienen un amplio potencial para generar bienestar en la población, son de difícil seguimiento debido precisamente a la ambigüedad y discrecionalidad con lo que los recursos y las acciones se desarrollan.

Por otra parte, es importante destacar que parece haber una desvinculación entre las instituciones participantes, así como entre las acciones que se les atribuyen, por ejemplo, el hecho de que no exista una adecuada especificidad de los trabajos que deben realizar las agencias federales y las instituciones locales en la Ciudad de México, en sus respectivos marcos de competencias, genera una disparidad institucional y un vacío de responsabilidad política y administrativa en lo que se refiere a los parques de bolsillo. Por otro lado, las agencias locales han fallado en establecer las reglas de operación de proyectos de esta naturaleza, así como las acciones de mantenimiento y vinculación con otros elementos urbanos tales como la movilidad, las actividades culturales y la seguridad.

Otro elemento importante a destacar lo constituyen los constantes cambios que en materia de administración pública se generan en México; lo anterior impacta de forma tal que un programa o proyecto que busca 
mejorar el nivel de vida de la población no se diseña considerando una visión administrativa y de acciones en el largo plazo. En general, existe una inadecuada continuidad de las políticas públicas y de una visión de largo plazo o de estrategias perennes. Lo anterior dificulta la planeación a mediano y largo plazo y, por tanto, condena al fracaso a proyectos que, en esencia, son loables. Sin embargo, a pesar de las ventajas y desventajas, las potencialidades y los desaciertos, no debemos dejar de lado que estas acciones constituyen importantes avances en materia de recuperación de espacios públicos.

Para que estos espacios subutilizados sean recuperados y tengan la aceptación social adecuada, es necesario llevar a cabo estrategias por parte de las autoridades de la Ciudad de México y las alcaldías, de forma tal que puedan ser promovidas las buenas prácticas de recuperación, uso y aprovechamiento de los espacios y se consideren áreas de disfrute para el beneficio barrial. Para esto, sigue siendo necesario rescatar de forma más extensa la opinión de los ciudadanos de estos espacios en ejercicios de investigación como los propuestos por este documento. Estos estudios deben ser ampliados y promovidos a fin de integrar la percepción que un número significativo de usuarios tengan respecto a estos parques; para ello es necesario, en principio, generar información precisa en torno al volumen de personas y las tendencias de uso que estos hacen.

Los indicadores aquí seleccionados, y los casos de estudio revisados, pueden y deben ser ampliados y enriquecidos a fin de considerar otras condiciones que influyan en el nivel de percepción de los usuarios al respecto de estos parques, así como de otras realidades en donde se instalen parques, de esta manera se conocerá mejor el entorno y el perfil de los beneficiarios de estas estrategias de recuperación.

Queda claro que el verdadero beneficio se observará cuando estos lugares realmente cumplan sus objetivos, den un valor agregado a la localidad y se tome en cuenta la opinión a propósito de mejoras por parte de los ciudadanos, también es importante que estos aspectos de mejora generen una identidad local para que los vecinos aumenten el valor de su zona en cuanto a imagen y promoción cultural, mientras eso sucede, los lugares recuperados corren el riesgo de convertirse en lugares olvidados o invisibles, con bajo impacto ambiental y social.

\section{Fuentes consultadas}

AEP (Autoridad del Espacio Público) (2018), "5to Informe de Gobierno", Ciudad de México, Autoridad del Espacio Público del Gobierno de la Ciudad de México,<http://www.aep.cdmx.gob.mx/storage/ 
app/uploads/public/59f/7dc/630/59f7dc63032d5263291804. pdf> 16 de mayo de 2018.

ALDF (Asamblea Legislativa del Distrito Federal) (2016), "Exhortan al Director General del Sistema de Aguas de la CDMX a renovar la red hidráulica de la U.H. El Rosario, Azcapotzalco", 28 de abril de 2016, boletín de prensa, <http://aldf.gob.mx/comsoc-exhortan-al-director-general-sistema-aguas-cdmx-renovar-red-hidraulica-u-h-rosario-azcapotzalco--25267.html>, 14 de enero de 2019.

Bellet Sanfeliu, Carmen y Llop Torné, Josep María (2004), "Miradas a otros espacios urbanos: las ciudades intermedias", Scripta Nova, Revista Electrónica de Geografía Ciencias Sociales, 8 (165), Barcelona, Universidad de Barcelona, pp. 741-798.

Borja, Jordi y Muxí, Zaida (2000), El espacio público, ciudady ciudadanía. Barcelona, Ed. Electa.

Bratina Jurkovic, Natasa (2014), "Perception, experience and the use of public urban spaces by residents of urban neighborhoods", Urbani Izziv. Urban Challenge Journal, 25 (1), Ljubljana, Urban Planning Institute of the Republic of Slovenia, pp. 107-125.

Carrión, Fernando (2016), "El espacio público es una relación, no un espacio", en Patricia Ramírez Kuri (coord.), La reinvención del espacio público en la ciudad fragmentada, Ciudad de México, Universidad Nacional Autónoma de México, pp. 13-47.

Checa-Artasu, Martín Manuel (2017), "De la percepción del paisaje a su consideración como derecho. El largo camino para el paisaje en México", Félix Alfonso Martínez Sánchez, Karla María Hinojosa de la Garza y Armando Alonso Navarrete (coords.), Arte, Historia y Cultura. Nuevas aproximaciones al conocimiento del paisaje, Ciudad de México, Universidad Autónoma Metropolitana unidad Azcapotzalco, pp. 165-188.

Clichevsky, Nora (2007), "La tierra vacante revisitada. Elementos explicativos y potencialidades de utilización”, Cuaderno Urbano. Espacio, cultura, sociedad, 6 (6), Chaco, 20 de noviembre de 2016, Universidad Nacional del Nordeste, pp. 195-219,<http://www. redalyc.org/articulo.oa?id=369236767010>, 
Curzio de la Concha, Claudio (2008), "El origen y las características de los fragmentos urbano-públicos residuales”, Cuadernos Geográfcos, núm. 42, Granada, Universidad de Granada, pp. 43-82.

Delgadillo, Víctor (2016), "Ciudad de México, disputas por el patrimonio urbano y el espacio público", en Patricia Ramírez Kuri (coord.), La reinvención del espacio público en la ciudad fragmentada, Ciudad de México, Universidad Nacional Autónoma de México, pp. 135-170.

Fausto, Adriana y Rábago, Jesús (2001), “¿Vacíos urbanos o vacíos de poder metropolitano?”, Revista Ciudades, 13 (49), Madrid, Instituto Juan de Herrera, pp. 1-12

GDF (Gobierno del Distrito Federal) (2011), "Plan Integral de Manejo del Centro Histórico de la Ciudad de México (2011-2016)", Ciudad de México, Autoridad del Centro Histórico.

Iracheta, Alfonso (2014), El suelo urbano en Iberoamérica: crisis y perspectivas, Zinacantepec, El Colegio Mexiquense A. C.

León Balza, Sergio (1998), “Conceptos sobre espacio público, gestión de proyectos y lógica social: reflexiones sobre la experiencia chilena”, Revista EURE, 24 (71), Santiago de Chile, Universidad Católica de Chile, pp. 27-36.

Quiroga, Gabriela (2017), Procesos para el uso del espacio público y la publicidad, Ciudad de México, Agencia del Espacio Público, <http://www.adocac.mx/web/images/descargas/congreso14/ EspacioPublicoyPublicidad.pdf>, 20 de mayo de 2018.

Rabotnikof, Nora (2010), “Discutiendo lo publico en México”, en Mauricio Merino (coord.), ¿Qué tan público es el espacio público en México?, Ciudad de México, Fondo de Cultura EconómicaConsejo Nacional de Cultura y las Artes-Universidad Veracruzana, pp. 25-56.

Sedema (Secretaría del Medio Ambiente) (s/f), "Parques y jardines", Ciudad de México, Gobierno de la Ciudad de México, <http:// data.sedema.cdmx.gob.mx/areasverdesvidaparatodos/parques_jardines.html\#.XOYSEhYzaUk> 20 de abril de 2018. 
Seduvi (Secretaría de Desarrollo Urbano y Vivienda) (2014), "Lineamientos para el diseño e implementación de parques públicos de bolsillo", Ciudad de México, Seduvi, <http://sistemadecalles. agucdmx.gob.mx/doctos/06.pdf >, 1 de abril de 2020 .

Sosa, Alejandro (2013), "Mancera inaugura el primer 'Parque Público de Bolsillo’ en el Zócalo capitalino”, Excélsior, 15 de abril, ciudad de México, Grupo Imagen, <https://www.excelsior.com.mx/comunidad/2013/04/15/894040>, 30 de abril de 2018.

Subirats, Joan, (2016), "Explorar el espacio público como bien común. Debates conceptuales y de gobierno en la ciudad fragmentada", en Patricia Ramírez Kuri (coord.), La reinvención del espacio público en la ciudad fragmentada Ciudad de México, Universidad Nacional Autónoma de México, pp. 73-98.

Terrazas-Revilla, Óscar (2005), La ciudad de los caminos: el caso del corredor Tlaxcala-Puebla, Ciudad de México, Universidad Autónoma Metropolitana unidad Azcapotzalco.

Terrazas-Revilla, Óscar (1995), "Los ejes de la metropolización”, Anuario de Estudios Urbanos, núm. 2, Ciudad de México, Universidad Autónoma Metropolitana unidad Azcapotzalco, pp. 317-339.

Yin, Robert (2009), Case study research, California, Sage.

Recibido: 22 de diciembre de 2019.

Reenviado: 26 de marzo de 2020. Aceptado: 6 de abril de 2020.

Laura Elena Martínez Salvador. Doctora en Economía por el Instituto de Investigaciones Económicas, Universidad Nacional Autónoma de México (UNAM), maestra en Economía y licenciada en Administración por la Universidad Autónoma Metropolitana. Miembro del Sistema Nacional de Investigadores nivel Candidato. Actualmente es investigadora asociada del Instituto de Investigaciones Sociales de la UNAM. Sus líneas de investigación son, en torno a la gobernanza y las políticas públicas, los sistemas agroalimentarios localizados y la protección institucional de la agrobiodiversidad. Entre sus últimas publicaciones destacan: como coautora, "Smart specialization for building up a regional innovation agenda: the case of San Luis Potosí, Mexico" Journal of Evolutionary Studies in 
Business, 5 (1), Barcelona, Universitat de Barcelona, pp. 81-115 (2020); coautora, "Los fallos de la política de innovación en México", Revista Debates Sobre Innovación, 2 (1), Ciudad de México, Universidad Autónoma Metropolitana unidad Xochimilco, pp. 1-22 (2019) y como autora "Capacidades tecnológicas en la agroindustria en México. Marco analítico para su investigación”, Revista Análisis Económico, 33 (84), Ciudad de México, Universidad Autónoma Metropolitana unidad Azcapotzalco, pp. 169-189.

David Fabricio Alvarado Ramírez. Maestro en Diseño y Estudios Urbanos y Diseñador Industrial por la Universidad Autónoma Metropolitana unidad Azcapotzalco, Actualmente es estudiante de Doctorado en Ciencias en Conservación del Patrimonio Paisajístico del Centro Interdisciplinario de Investigaciones y Estudios sobre Medio Ambiente y Desarrollo del Instituto Politécnico Nacional. Sus líneas de investigación se han desarrollado en torno al desarrollo urbano y periurbano, los sistemas productivos locales y el desarrollo territorial. Entre sus últimas publicaciones destacan: como coautor, "Protección institucional de la agrobiodiversidad en América Latina. Un análisis desde los sistemas agroalimentarios localizados de quínoa boliviana y amaranto mexicano", en Marie-Christine Renard y Jessica Mariela Tolentino Martínez (coords.), Red SIAL México Diez años de contribución a los estudios de los Sistemas Agroalimentarios Localizado, Ciudad de México, Consejo Nacional de Ciencia y Tecnología-Red de Sistemas Agroalimentarios Localizados-Instituto Interamericano de Cooperación para la Agricultura-Yod Estudio, pp. 165-184 (2019); como coautor, "Agroindustria para el desarrollo de los territorios rururbanos. Una propuesta de análisis desde el huautli en la Ciudad de México", en Jessica Mariela Tolentino Martínez, Rosa María Larroa Torres, Marie-Christine Renard Hubert y Maria del Carmen del Valle Rivera (coords,), Sistemas Agroalimentarios Localizados y prácticas agricolas tradicionales. Hacia una propuesta de política pública para el desarrollo rural, Ciudad de México, Consejo Nacional de Ciencia y Tecnología-Red de Sistemas Agroalimentarios Localizados-Instituto Interamericano de Cooperación para la Agricultura-Yod Estudio, pp. 101-123 (2018). 